\title{
Systematic review and meta-analysis of various risk factors of death from emergency cerebrovascular diseases in the department of neurology
}

\author{
Yuanchao Zhang", Hang Yü, Chengming Ke, Qiaosong Sun \\ Neurology Medicine Center, The Seventh Affiliated Hospital of Sun Yat-sen University, Shenzhen, China \\ Contributions: (I) Conception and design: Y Zhang, H Yu; (II) Administrative support: C Ke; (III) Provision of study materials or patients: Y Zhang, H \\ Yu, Q Sun; (IV) Collection and assembly of data: All authors; (V) Data analysis and interpretation: Y Zhang, C Ke, Q Sun; (VI) Manuscript writing: \\ All authors; (VII) Final approval of manuscript: All authors. \\ \#These authors contributed equally to this work. \\ Correspondence to: Yuanchao Zhang. Neurology Medicine Center, The Seventh Affiliated Hospital of Sun Yat-sen University, 628 Zhenyuan Road, \\ Guangming District, Shenzhen, China. Email: zhangyuanchao6938@163.com.
}

Background Cerebrovascular disease is one of the leading causes of death and disability in China. Preventive measures to reduce the incidence of cerebrovascular disease are important, so the risk factors associated with death need to be identified. Most studies showed that cerebrovascular disease has many risk factors for death, such as hypertension, hyperlipidemia, family history of stroke, diabetes, overweight, alcohol consumption, and smoking.

Methods: A literature search was conducted in the English database PubMed and Chinese databases including CNKI, VIP, and China Journal Full-text Database. The time limit for retrieval was from establishment of the database to March 2021. All randomized controlled trials (RCTs) involving hypertension, hyperlipidemia, family history of stroke, diabetes, overweight, drinking, smoking and cerebrovascular diseases (i.e., stroke and cerebral infarction) were included. Review Manager 5.3 provided by the Cochrane Collaboration was used for meta-analysis.

Results: A total of 10 studies (with 32,664 patients in trial and control groups) were included: 14,743 cases in the control group and 17,901 cases in the risk factor group. The combined odds ratio (OR) and $95 \%$ confidence interval $(95 \% \mathrm{CI}$ ) of all risk factors in patients with emergency cerebrovascular diseases in the Department of Neurology were 2.33 (1.83-2.98) for hypertension, 2.65 (1.80-3.91) for hyperlipidemia, 2.18 (1.73-2.73) for family history of stroke, 4.78 (3.07-7.42) for overweight, 2.97 (1.95-4.52) for alcoholism, and 2.98 (2.11-4.2) for smoking. P values were all less than 0.05 , and the differences were statistically significant.

Discussion: The 10 articles included in this meta-analysis studied the effects of various mortality risk factors (hypertension, hyperlipidemia, family history of stroke, diabetes, overweight, alcohol consumption, and smoking) on the death of patients with emergency cerebrovascular diseases in the Department of Neurology. Attention should be paid to the treatment or care of the above factors in clinical practice to reduce the mortality of patients.

Keywords: Cerebrovascular disease; death risk factors; meta-analysis

Submitted Aug 08, 2021. Accepted for publication Sep 24, 2021.

doi: 10.21037/apm-21-2329

View this article at: https://dx.doi.org/10.21037/apm-21-2329 


\section{Introduction}

Cerebrovascular disease is one of the leading causes of death and disability in China (1). According to the statistics of the Ministry of Health in Beijing in 1999, the mortality rate of cerebrovascular diseases in China is 137 per 100,000 people (2). Preventive measures to reduce the incidence of cerebrovascular disease are important, so this study aimed to identify the risk factors associated with death. Most studies show that cerebrovascular disease has many fatal risk factors, and chronicity is an independent risk factor of cerebrovascular disease (3). The age of patients at onset of acute cerebrovascular disorders has shown a tendency to become younger. The results reported by Barthels and Das (4) indicated an increase in the prevalence of cerebrovascular diseases in emergency departments in developing countries over the past 10 years compared with previous decades. Chronic diseases such as hypertension are a risk factor of cerebrovascular diseases. People with diabetes have a high risk of cerebrovascular disease. Among patients with cerebrovascular diseases (e.g., stroke and cerebral infarction), the number of patients with diabetes is twice that of those without diabetes (5). Most of the patients with cerebral infarction and stroke cannot use anti-glycemic drugs because they shorten the onset of hypertension, hyperlipidemia, and hyperglycemia, so the patients are not optimally treated, forming a vicious circle. Being overweight may also be a risk factor for death from cerebrovascular disease. Zheng et al. (6) tracked 300 older adults in 2001, 47 of whom had hypertension, and 79 cases of cerebrovascular diseases were tracked over a 6-year period. The annual incidence of cerebrovascular disease in the obese patients was $3.77 \%$. Other studies have shown that alcohol intake is also greatly related to cerebrovascular diseases, and the incidence of disease is related to the frequency and amount of alcohol intake (7). In addition, there is a significant association between alcohol intake and the incidence of intracerebral hemorrhage and subarachnoid hemorrhage, but no significant association between alcohol intake and ischemic stroke. Smoking is also a risk factor for cerebrovascular disease (8).

The purpose of this systematic review was to summarize the evidence from randomized controlled trials (RCTs) designed to prevent deaths from cerebrovascular disease in the emergency department. The risk of deviation in quality, quantity, and treatment effectiveness was compared. The inherent focus of this approach was studying the association between risk factors and various cerebrovascular diseases, rather than to compare their therapeutic effects. Different cerebrovascular diseases have different treatments, but the risk factors are mostly similar for interventions that work in very different ways. In this study, to discuss the various risk factors for death from emergency cerebrovascular diseases in the Department of Neurology, a total of 10 RCTs were included (9-18). The results of comparative studies of domestic and foreign cases were reviewed, and various screening and meta-analyses were carried out. It provides the scientific basis and theoretical basis for reducing the mortality rate of emergency cerebrovascular diseases and preventing the recurrence of cerebrovascular diseases in the neurology department. The innovation of this article lies in the use of meta-analysis of various death risk factors for emergency cerebrovascular diseases in the Department of Neurology, and a collection of 10 documents to verify the results, which including hypertension, risk factors for death, hyperlipidemia, family history of stroke, diabetes, overweight, and alcohol consumption. The effect of smoking on cerebrovascular diseases has been studied, it not only includes stroke, but also includes ischemic brain diseases and cerebral infarction, to improve the diagnosis rate and cure rate of patients. We present the following article in accordance with the PRISMA reporting checklist (available at https://dx.doi.org/10.21037/apm-21-2329).

\section{Methods}

\section{Literature search strategy}

Medline, Embase, and the Cochrane Central Register of Controlled Trials were searched for postoperative pulmonary complications related keywords and a combination of medical subject terms. The search restriction limited the results to the published RCT studies included in each database from the beginning of the construction of the database until March 2021. The selected start date overlapped with the last systematic review of prevention strategies. Descriptive terms were combined with migraine, smoking, history of chronic disease, paralysis, infection, hypotension, hyponatremia, insomnia or stress, diabetes, dyslipidemia, smoking, small vessel disease and infarction, risk factors for death, cerebrovascular disease, cerebral infarction, and stroke. Chinese search keywords included migraine, smoking, history of chronic diseases, paralysis, infection, hypotension, hyponatremia, insomnia or stress, diabetes, dyslipidemia, smoking, small vessel disease infarction, death risk factors, cerebrovascular 
disease, brain infarction, and stroke. According to preestablished inclusion and exclusion criteria, the full text of the target study was obtained. In addition, the symptoms and treatments of cerebrovascular diseases were also studied.

\section{Literature inclusion and exclusion criteria}

Inclusion criteria: (I) patients diagnosed with CT or MRI according to a revised diagnostic basis of the 4th National Diagnostic Conference on Cerebrovascular Diseases or WHO diagnostic baseline; (II) patients older than 18 years; (III) patients with cerebrovascular disease who were treated in the emergency department; (IV) patients had a prior history of stroke, cerebral infarction, or other cerebrovascular disease; (V) trials had both experimental and control groups.

Exclusion criteria: (I) unclear data; (II) trials lacking required disease data; (III) no controlled trial; (IV) results were not credible.

\section{Literature screening}

Independent literature screening and data extraction were performed, and finally cross-examination was done. If different opinions arose, experts of Neurology Emergency Physician were consulted to discuss the data selection.

\section{Data extraction}

Data extraction in this study was carried out independently by two researchers. In the process of data extraction, the researchers independently extracted the study data first, creating an Excel table based on the basic information, characteristics of the research subjects, intervention measures, outcome indicators, and bias evaluation data. After the extraction, cross-checking was conducted. If there were any differences in opinion, discussion and settlement could be made between the two researchers, or opinions could be sought from a third researcher. In this study the data to be extracted included basic literature information (title, first author, publication year, author information, and literature source), basic characteristics of the subjects (sex, age, study sample size, and baseline comparability), study method, study scheme design, intervention measures of experimental and control groups, outcome evaluation indicators, and outcome data.

\section{Quality evaluation}

To improve the quality of the reviewed literature, quality was assessed in accordance with the "risk of bias assessment" recommended in version 5.3 of the Cochrane system review manual. The evaluation included the following seven items: (I) which random method was used; (II) whether there was hidden allocation; (III) the implementation of blinding method between patients and researchers; (IV) evaluation of the effect of the blinding method; (V) whether there was the integrity of the results; (VI) whether the survey results were credible; (VII) other biases regarding each RCT in item VI above. "Satisfied" meant there was a small bias, "dissatisfied" meant there was a large bias and not enough detail to report it. If not mentioned, the bias was unknown. The assessment included four dimensions of random sequence generation, assignment concealment, blinding, and trace/exit. A score of 1-3 was considered low quality, and 4-7 was considered high quality.

\section{Statistical methods}

Review Manager 5.3 provided by the Cochrane Collaboration was used for meta-analyses. Odds ratio (OR) was used as the effect size, and the outcome was represented by $95 \%$ confidence interval (CI). First, the included studies were tested for heterogeneity, and $\alpha=0.1$ was taken as the test level. If there was no heterogeneity among studies $\left(\mathrm{P}>0.1, \mathrm{I}^{2}<50 \%\right)$, a fixed-effect model was selected for the meta-analysis. Otherwise, subgroup analysis was performed on the included data. $\mathrm{P}<0.05$ was considered statistically significant. When the number of references included in the analysis of a single risk factor was $>10$, funnel plots were used to analyze the publication bias of each risk factor.

\section{Results}

\section{Literature screening results}

A total of 210 articles were collected and based on the inclusion and exclusion criteria, a total of 10 articles (6-15) were included in this meta-analysis (Figure 1). The studies were published in seven journals from 20 hospitals in three countries, with a total of 14,743 cases and 17,901 control cases. The 10 articles (6-15) were obtained from PubMed and comprised the following: 10 studies of hypertension and stroke (6-15), 10 studies of hyperlipidemia and stroke (6-15), 9 studies of family history of stroke and stroke 


\section{Identification of studies via databases and registers}

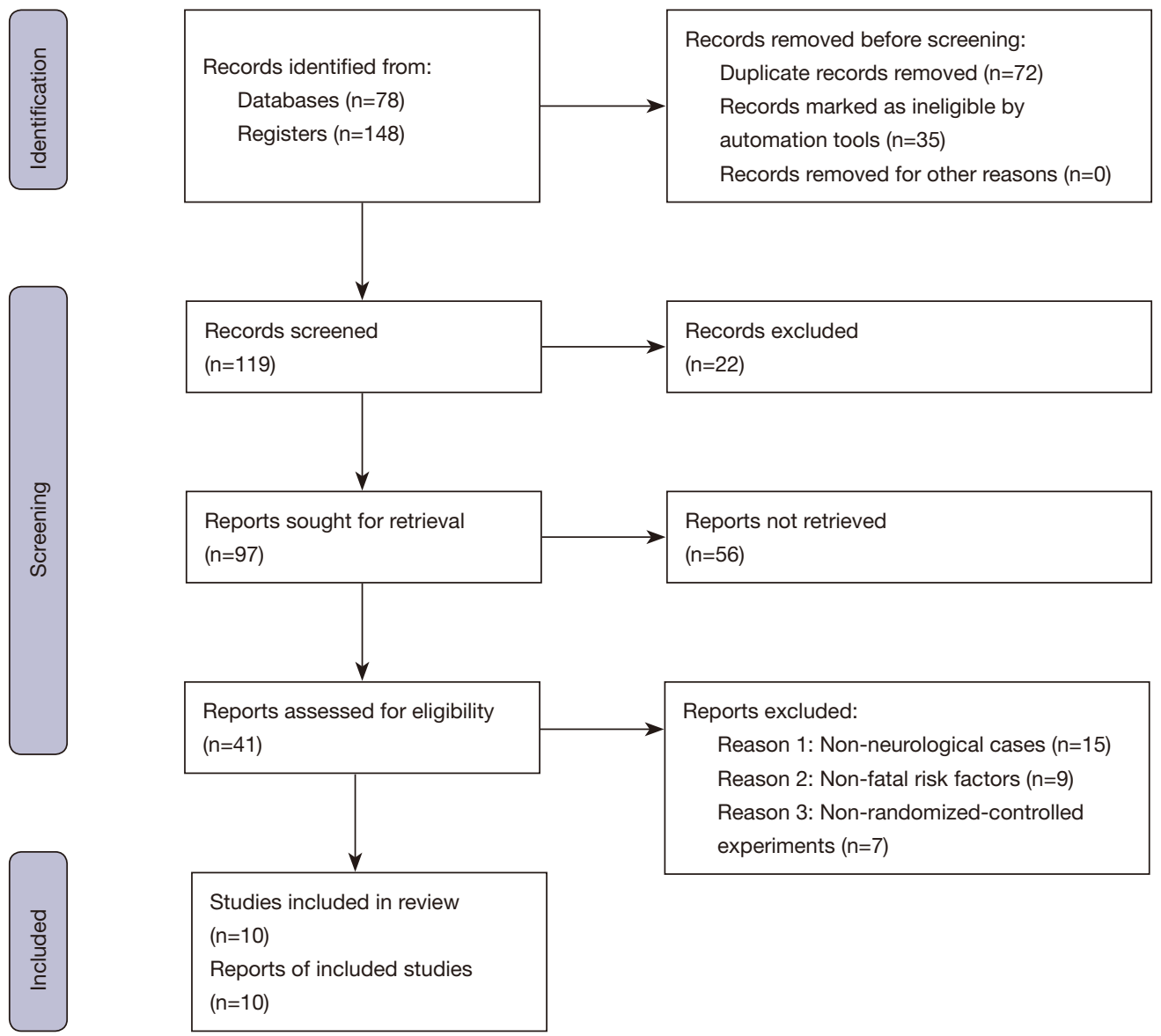

Figure 1 Literature screening process.

(6-14), 6 studies of diabetes and stroke (6-9,12-15), 6 studies of overweight and stroke (6-8,11-15), 8 studies of drinking and stroke (6-11,12-14), and 7 studies of smoking and stroke $(6-11,13,14)$ (Table 1).

\section{Results of risk bias evaluation of included studies}

Using Review Manager 5.3's risk of bias assessment tool to evaluate the quality of the included 10 articles, the results were shown in Figure 2 and Figure 3. Most aspects of the included RCTS were assessed as having a low risk of bias. Due to the characteristics of face masks used outside the body, it is difficult to blind researchers and subjects during this process. Therefore, the blindness (performance bias) of the participants and personnel included in this study was assessed as high risk. Based on the completeness of the data and the selectivity of the report, it is classified as low risk.

\section{Hypertension and emergency cerebrovascular disease in the neurology department}

Meta-analysis was performed on 10 RCTs obtained for middle-aged, elderly, and general populations through the literature screening process. "Hypertension" was used as exposure in the analysis, while "non-hypertensive" cases were included in the control group. The exposure/nonexposure definitions in all RCTs were similar. In the 10 studies, a total of 17,149 patients were included. After heterogeneity testing, the results showed that $\mathrm{I}^{2}=91 \%$ and $\mathrm{P}<0.00001$, indicating that significant heterogeneity in the literature, so the random effects model was used for analysis. Meta-analysis results showed that $\mathrm{OR}=2.33$, 
Table 1 Basic data of included studies

\begin{tabular}{|c|c|c|c|c|c|}
\hline First author & Year & $\begin{array}{l}\text { Types of } \\
\text { cerebrovascular } \\
\text { diseases }\end{array}$ & Risk factors & Age (years) & $\begin{array}{l}\text { Total } \\
\text { patients/ } \\
\text { case }\end{array}$ \\
\hline Li (10) & 2015 & Stroke & $\begin{array}{l}\text { Great artery disease, small vessel disease, cardiac } \\
\text { embolism, atherosclerosis, diabetes, peripheral } \\
\text { vascular disease, hypercholesterolemia, smoking, heart } \\
\text { disease, smoking, alcohol consumption, hyperglycemia, } \\
\text { hyperlipidemia }\end{array}$ & $>18$ & 2,555 \\
\hline Bowers (12) & 2006 & Stroke & $\begin{array}{l}\text { Brain tumor, cerebral palsy, heart disease, smoking, } \\
\text { drinking, hyperglycemia, hyperlipidemia }\end{array}$ & $>18$ & 100 \\
\hline MacClellan (13) & 2007 & Stroke & $\begin{array}{l}\text { Smoking, oral contraceptive, age, hypertension, diabetes, } \\
\text { angina pectoris, smoking, migraine }\end{array}$ & $15-49$ & $386+614$ \\
\hline O'Donnell (14) & 2010 & Stroke & $\begin{array}{l}\text { Age, hyperlipidemia, smoking, diabetes mellitus, } \\
\text { hypertension, abdominal obesity, heart disease, smoking, } \\
\text { alcohol consumption, hyperglycemia, hyperlipidemia }\end{array}$ & $>18$ & 3,000 \\
\hline Topcuoglu (15) & 2017 & Stroke & $\begin{array}{l}\text { Migraine, paralysis, infection, hypotension, hyponatremia, } \\
\text { insomnia or stress, diabetes, dyslipidemia, smoking, small } \\
\text { vessel disease, infarction }\end{array}$ & $>18$ & 153 \\
\hline Dupont (18) & 2009 & $\begin{array}{l}\text { Cerebral infarction } \\
\text { and stroke }\end{array}$ & $\begin{array}{l}\text { Age, hyperlipidemia, smoking, diabetes, hypertension, } \\
\text { abdominal obesity, heart disease, smoking, alcohol } \\
\text { consumption, hyperglycemia, hyperlipidemia }\end{array}$ & $>18$ & 208 \\
\hline
\end{tabular}

95\% CI: $1.83-2.98, Z=6.84$, and $\mathrm{P}<0.00001$, so there was a significant difference in postoperative mortality rates between the two groups $(\mathrm{P}<0.05)$. Figure 4 shows the forest plot.

The funnel plot was basically symmetric and most of the data corresponded to points within the 95\% CI (Figure 5). The non-safe number was 4,469 , indicating that at least 4,469 non-positive control results were required in order for the results associated with hypertension and cerebrovascular disease to be above $5 \mathrm{k}+10=95$, suggesting that publication bias was effective.

\section{Hyperlipidemia and emergency cerebrovascular diseases in neurology}

Meta-analysis was performed on 10 RCTs obtained for middle-aged, elderly, and general populations through the literature screening process. "Hypertension" was used as exposure in the analysis, while "non-hypertensive" cases were included in the control group. The exposure/nonexposure definitions in all literature studies were similar. In the 10 studies, 17,339 patients with cerebrovascular disease and 17,567 controls were included. After heterogeneity 


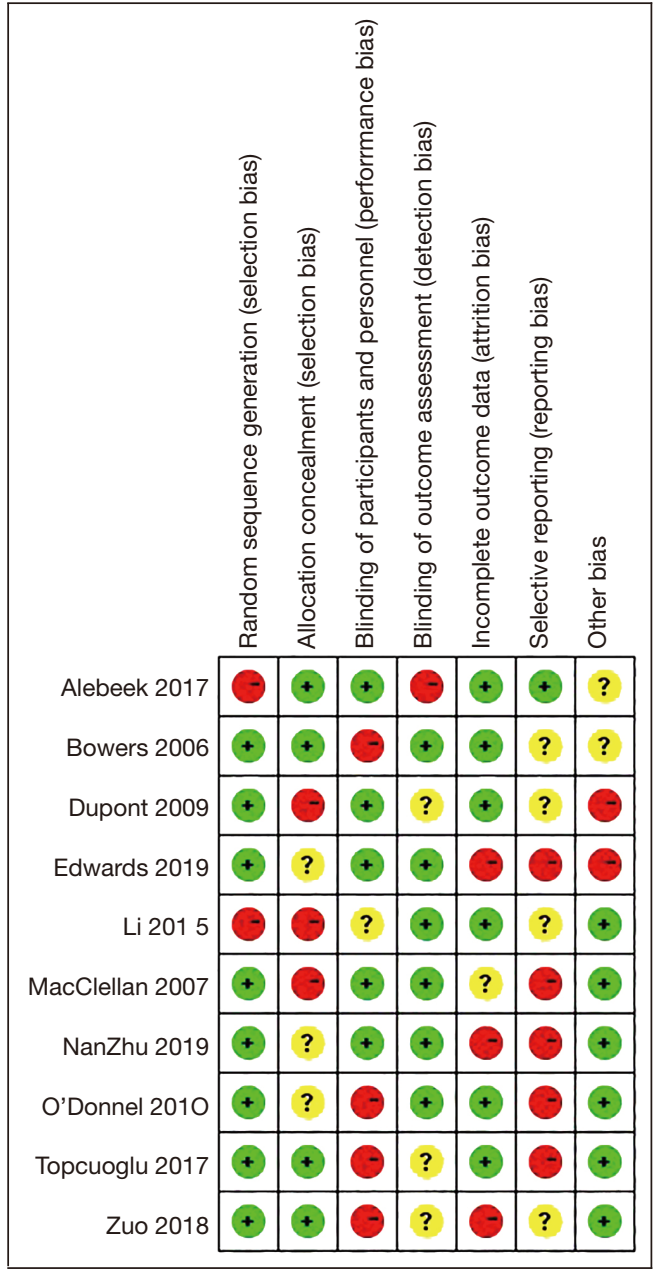

Figure 2 Literature risk bias evaluation results. detection, the results showed that $\mathrm{I}^{2}=97 \%$ and $\mathrm{P}<0.00001$, indicating significant heterogeneity in the literature, so the random effects model was used for analysis. Metaanalysis results showed that $\mathrm{OR}=2.65,95 \% \mathrm{CI}: 1.80-3.91$, $\mathrm{Z}=4.92$, and $\mathrm{P}<0.00001$, so there was a significant difference in postoperative mortality rates between the two groups $(\mathrm{P}<0.05)$. Figure 6 shows the forest plot.

The funnel plot was basically symmetric and most of the data corresponded to points within the $95 \%$ CI (Figure 7). The non-safe number was 4,469 , indicating that at least 4,469 non-positive control results were required in order for the results associated with hypertension and cerebrovascular disease to be above $5 \mathrm{k}+10=95$, suggesting that publication bias was effective.

\section{Family bistory of stroke and emergency cerebrovascular disease in neurology}

Meta-analysis was performed on 9 RCTs obtained for middle-aged, elderly, and general populations through the literature screening process. "History of stroke" was used as exposure in the analysis, while "non-history of stroke" cases were studied in the control group. The exposure/ non-exposure definitions in all studies were similar. In the 9 studies, a total of 5,585 patients were included. After heterogeneity testing, the results showed that $\mathrm{I}^{2}=85 \%$ and $\mathrm{P}<0.00001$, indicating significant heterogeneity in the literature, so the random effects model was used for analysis. Meta-analysis results showed that $\mathrm{OR}=2.18,95 \%$ CI:

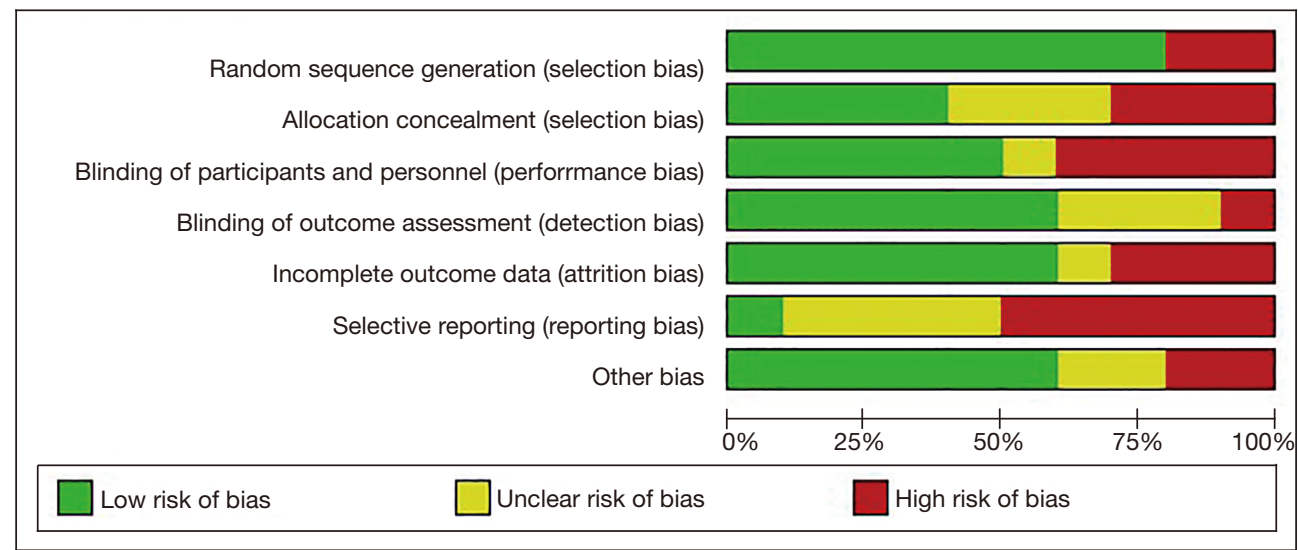

Figure 3 Literature bias risk assessment bar chart. 


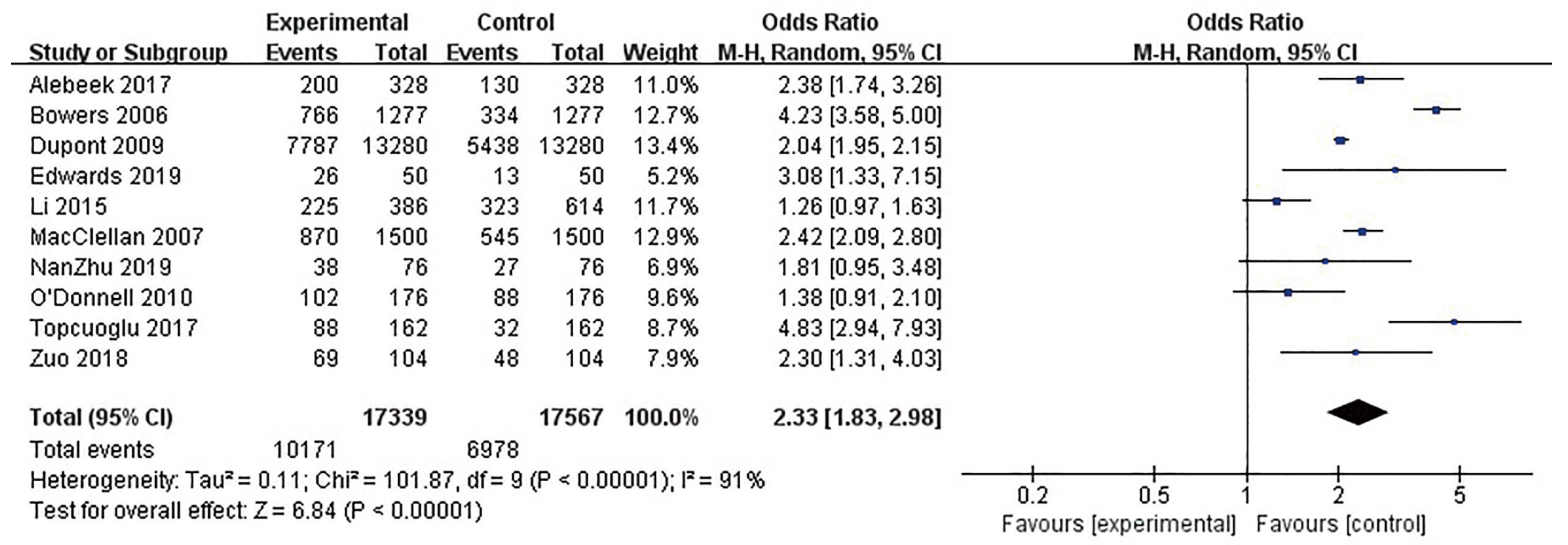

Figure 4 Forest plot of mortality rates of hypertension and cerebrovascular disease in the two groups.

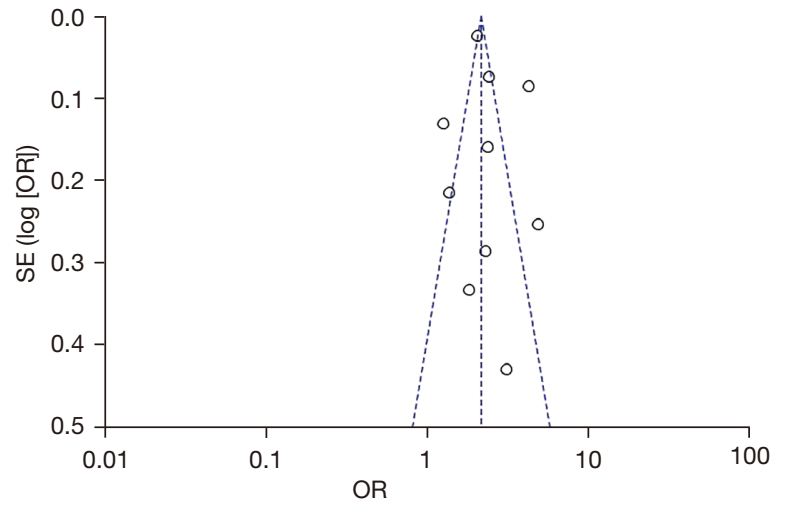

Figure 5 Funnel plot of mortality rates of hypertension and cerebrovascular disease in the two groups. SE, standard error; OR, odds ratio.
$1.73-2.73, \mathrm{Z}=6.70$, and $\mathrm{P}<0.00001$, so there was a significant difference in postoperative mortality rates between the two groups $(\mathrm{P}<0.05)$. Figure 8 shows the forest plot.

The funnel plot was basically symmetric and most of the data corresponded to points within the $95 \%$ CI (Figure 9). The non-safe number was 5,769, indicating that at least 5,769 non-positive control results were required in order for the results associated with stroke history and cerebrovascular disease to be above $5 \mathrm{k}+10=95$, suggesting that publication bias was effective.

\section{Overweight and emergency cerebrovascular disease in neurology}

Meta-analysis was performed on 6 RCTs obtained for

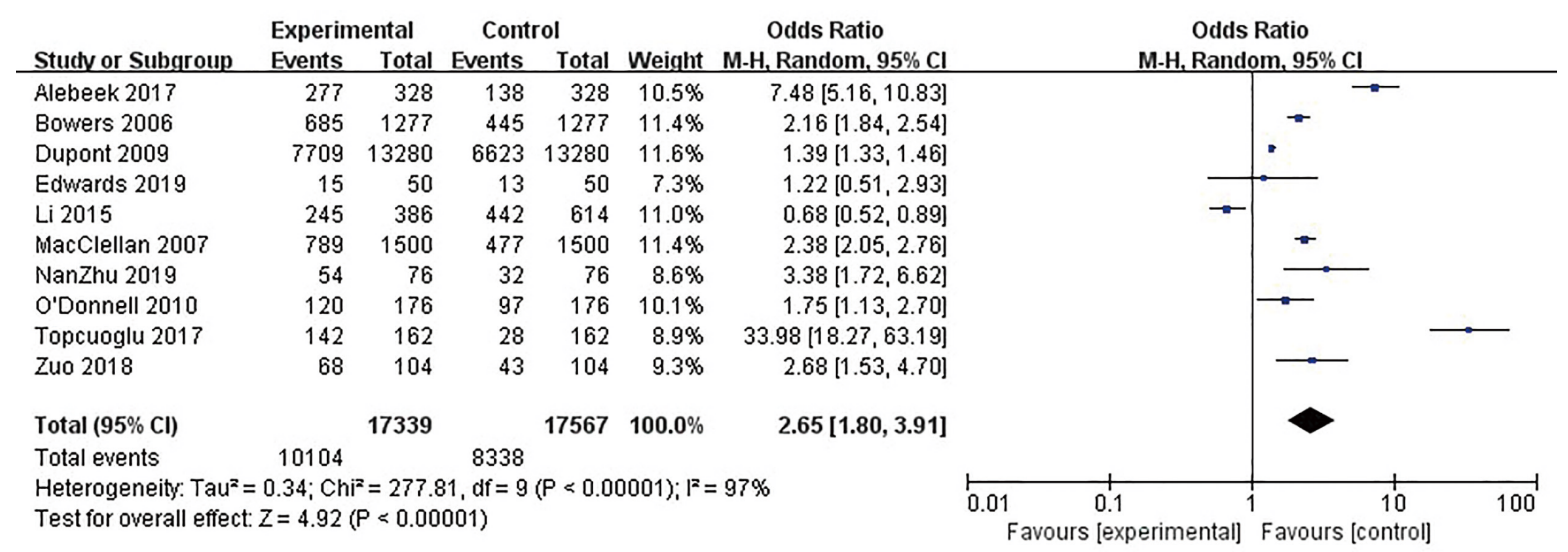

Figure 6 Forest plot of mortality rates of hyperlipidemia and cerebrovascular disease in the two groups. 
middle-aged, elderly, and general populations through the literature screening process. "Overweight" was counted as exposure in the analysis, while "non-overweight" cases were studied in the control group. The exposure/nonexposure definitions in all studies were similar. In the 6 studies, 17,339 patients with cerebrovascular disease and 17,567 controls were included. After heterogeneity testing, the results showed that $\mathrm{I}^{2}=97 \%$ and $\mathrm{P}<0.00001$, indicating significant heterogeneity in the literature, so the random effects model was used for analysis. Metaanalysis results showed that $\mathrm{OR}=4.78,95 \% \mathrm{CI}: 3.07-7.42$, $\mathrm{Z}=6.95$, and $\mathrm{P}<0.00001$, so there was a significant difference in postoperative mortality rates between the two groups $(\mathrm{P}<0.05)$. Figure 10 shows the forest plot.

The funnel plot was basically symmetric and most of the data corresponded to points within the $95 \%$ CI

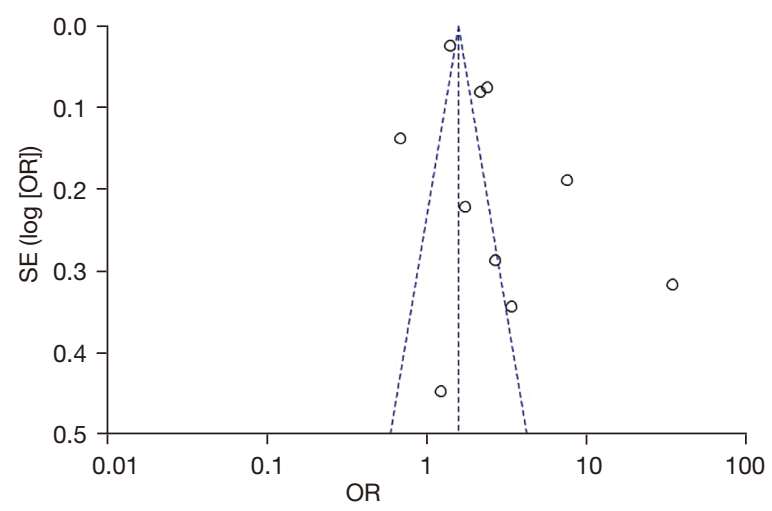

Figure 7 Funnel plot of mortality rates of hyperlipidemia and cerebrovascular disease in the two groups. SE, standard error; OR, odds ratio.
(Figure 11). The non-safe number was 5,698, indicating that at least 5,698 non-positive control results were needed for the correlation between overweight and cerebrovascular disease to be above $5 \mathrm{k}+0=95$, suggesting that publication bias was effective.

\section{Alcobol consumption and emergency cerebrovascular disease in neurology}

Meta-analysis was performed on 8 RCTs obtained for middle-aged, elderly, and general populations through the literature screening process. "Drinking" was used as exposure in the analysis, while "non-drinking" cases were studied in the control group. The exposure/non-exposure definitions in all studies were similar. In 8 studies, a total of 21,356 patients were included. After heterogeneity

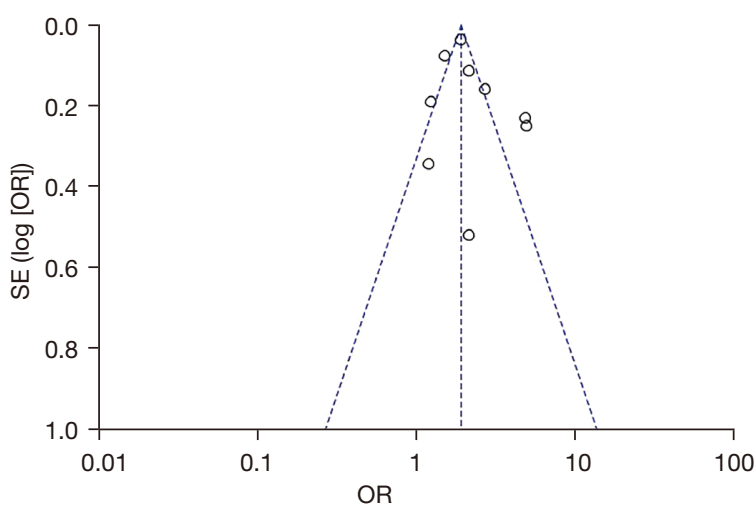

Figure 9 Funnel plot of stroke history and mortality rates of cerebrovascular disease in the two groups. SE, standard error; OR, odds ratio.

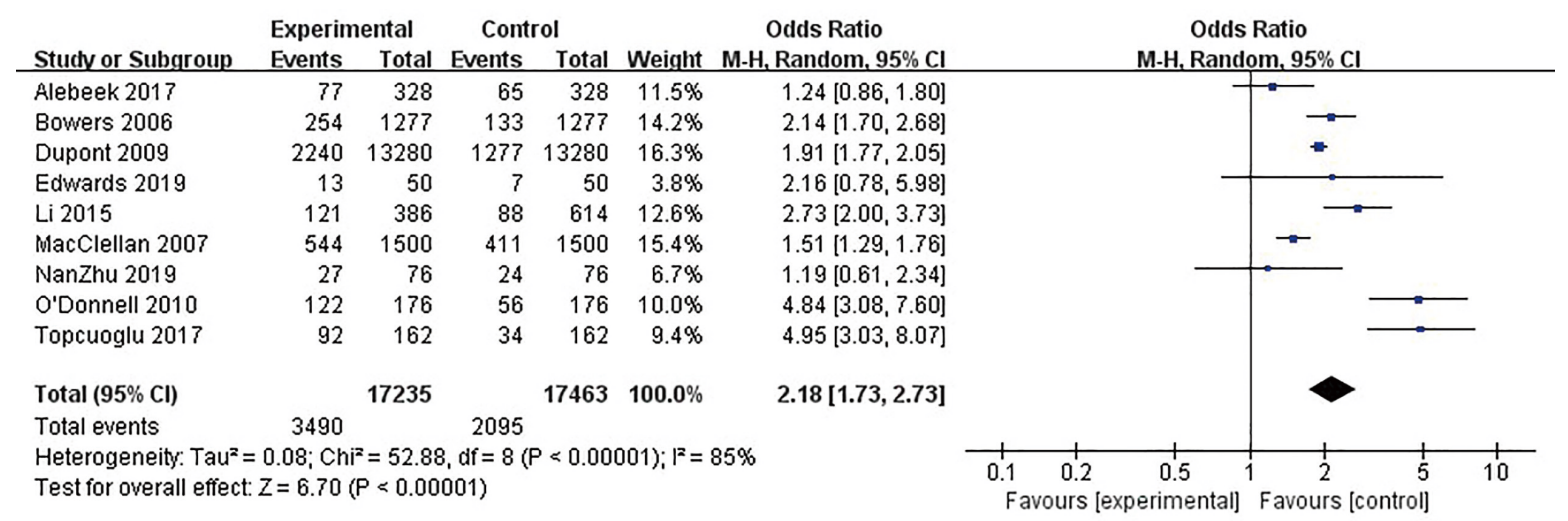

Figure 8 Forest plot of stroke history and mortality rates of cerebrovascular disease in the two groups. 


\begin{tabular}{lrrrrrrrr} 
& \multicolumn{1}{c}{$\begin{array}{c}\text { Experimental } \\
\text { Studv or Subgroup }\end{array}$} & $\begin{array}{c}\text { Cvents } \\
\text { Total }\end{array}$ & Events & Total & Weight & M-H, Random, 95\% Cl & \multicolumn{2}{c}{ Odds Ratio } \\
M-H, Random, 95\% Cl
\end{tabular}

Figure 10 Forest plot of overweight and mortality rates of cerebrovascular disease in the two groups.

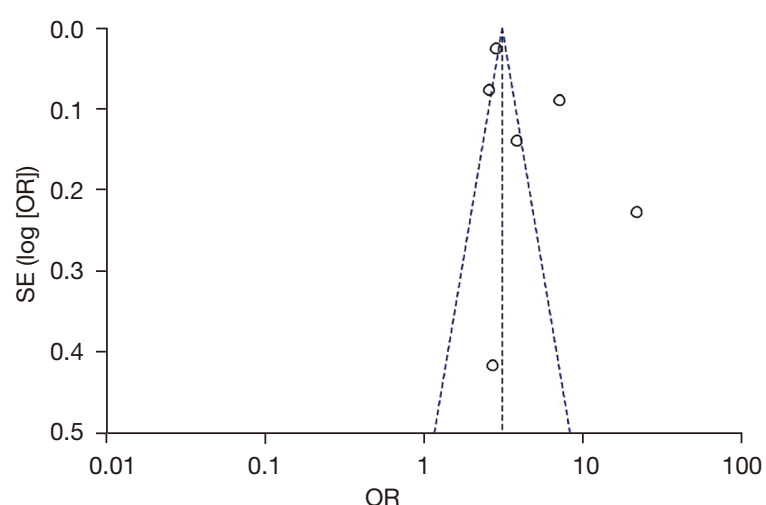

Figure 11 Funnel plot of overweight and mortality rates of cerebrovascular disease in the two groups. SE, standard error; OR, odds ratio. testing, the results showed that $\mathrm{I}^{2}=95 \%$ and $\mathrm{P}<0.00001$, indicating significant heterogeneity in the literature, so the random effects model was used for analysis. Metaanalysis results showed that $\mathrm{OR}=2.97,95 \% \mathrm{CI}$ : $1.95-4.52$, $\mathrm{Z}=5.07$, and $\mathrm{P}<0.00001$, so there was a significant difference in postoperative mortality rates between the two groups $(\mathrm{P}<0.05)$. Figure 12 shows the forest plot.

The funnel plot was basically symmetric and most of the data corresponded to points within the $95 \% \mathrm{CI}$ (Figure 13). The non-safe number was 6,788, indicating that at least 6,788 non-positive control results were needed for the association between alcohol consumption and cerebrovascular disease to be above $5 \mathrm{k}+10=95$, suggesting that publication bias was effective.

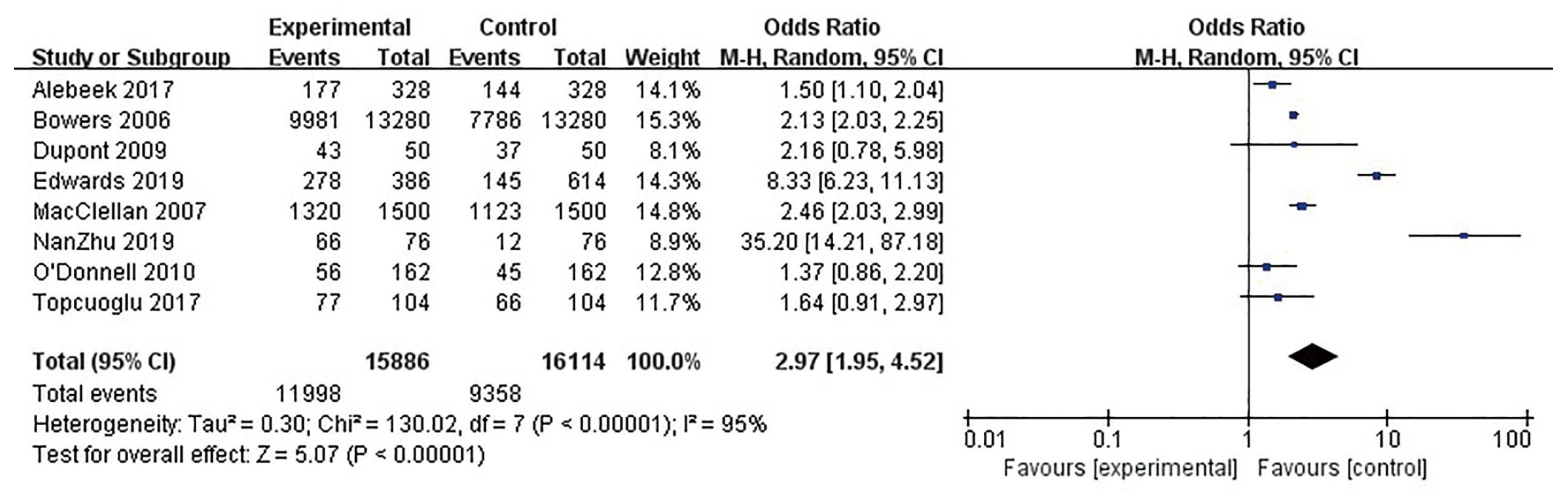

Figure 12 Forest plot of alcohol consumption and mortality rates of cerebrovascular disease in the two groups. 


\section{Smoking and emergency cerebrovascular diseases in neurology}

Meta-analysis was performed on 7 RCTs obtained for middle-aged, elderly, and general populations through the literature screening process. "Smoking" was taken as an exposure in the analysis. The seven studies included 17,339 patients with cerebrovascular disease and 17,567 controls. After heterogeneity testing, the results showed that $\mathrm{I}^{2}=85 \%$ and $\mathrm{P}<0.00001$, indicating significant heterogeneity in the literature, so the random effects model was used for analysis. Meta-analysis results showed that $\mathrm{OR}=2.98,95 \%$ CI: 2.11 $4.20, \mathrm{Z}=6.22$, and $\mathrm{P}<0.00001$, so there was a significant difference in postoperative mortality rates between the two groups $(\mathrm{P}<0.05)$. Figure 14 shows the forest plot.

The funnel plot was basically symmetric, and most of the data corresponded to points within the $95 \%$ CI

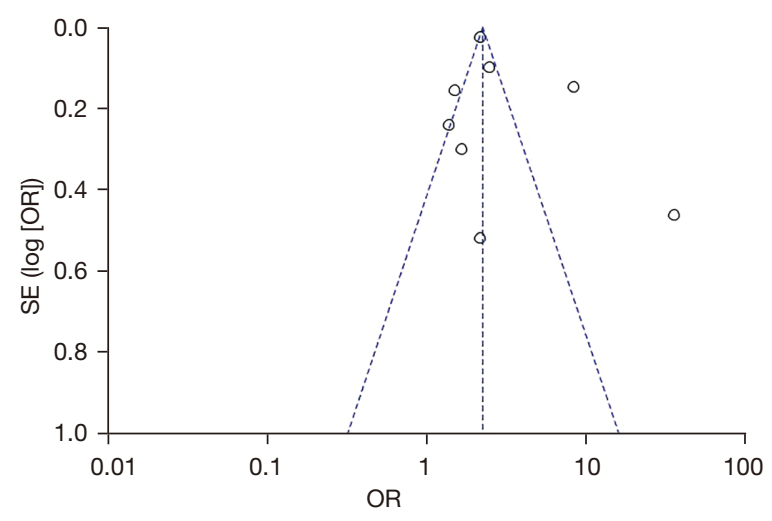

Figure 13 Funnel plot of alcohol consumption and mortality rates of cerebrovascular disease in the two groups. SE, standard error; $\mathrm{OR}$, odds ratio.
(Figure 15). The non-safe number was 7,756, indicating that at least 7,756 non-positive control results were required in order for the correlation between smoking and cerebrovascular disease to be above $5 \mathrm{k}+10=95$, suggesting that publication bias was effective.

\section{Discussion}

A total of 10 RCTs (6-15) were included in this metaanalysis. Among the quality evaluations of the studies, there were one paper of grade A quality and nine grade B papers. Among the 10 selected studies, six (6-11) reported basic data such as age, disease type, and disease stage. However, there was no statistically significant difference between the experimental and control groups $(\mathrm{P}>0.05)$. One study (15) used a table of random numbers for

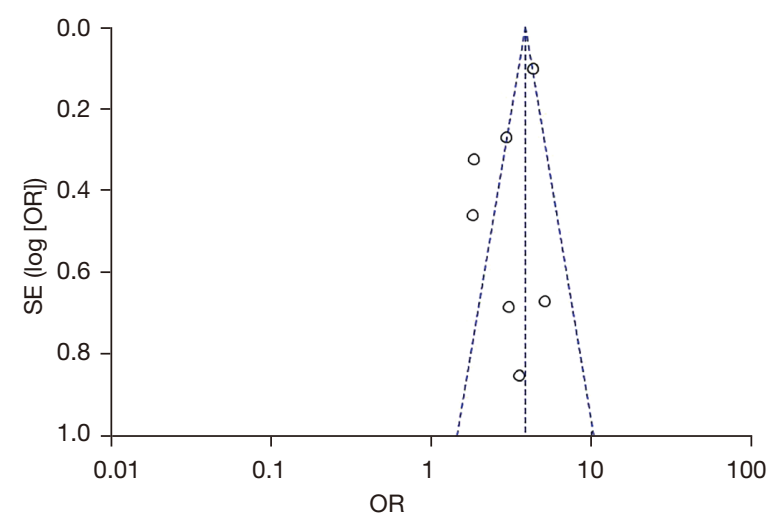

Figure 15 Funnel plot of smoking and mortality rates of cerebrovascular disease in the two groups. SE, standard error; OR, odds ratio.

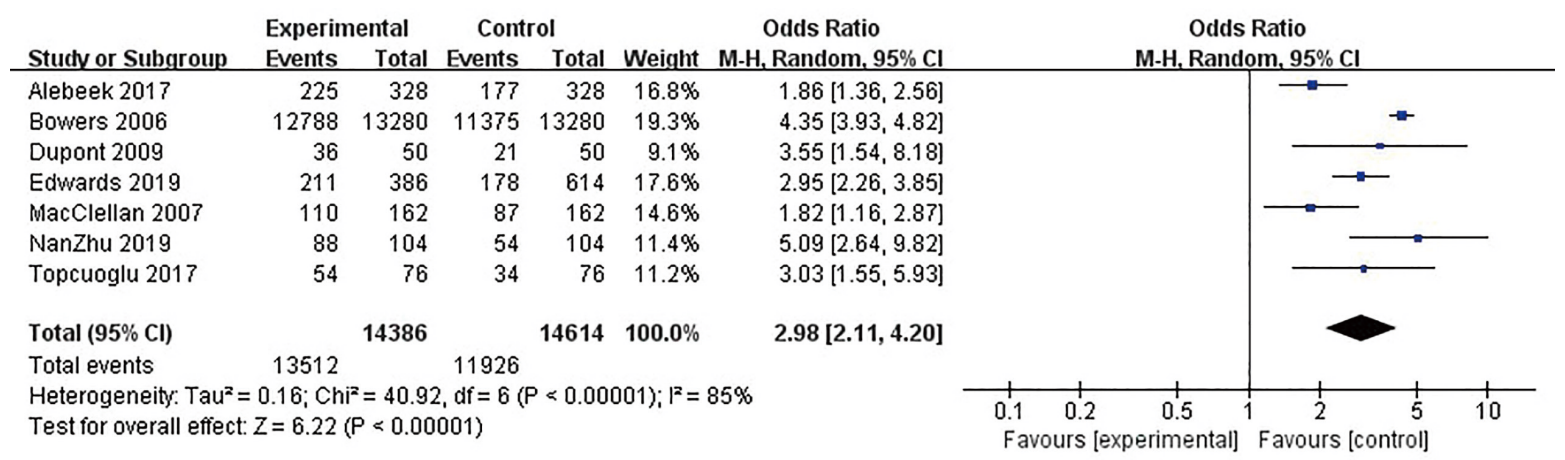

Figure 14 Forest plot of smoking and mortality rates of cerebrovascular disease in two groups. 
random combinations, and one study (13) was randomized according to treatment duration and treatment response. All 10 studies (6-15) introduced random methods, but did not report measurement blindness, nor did they report loss of observation process. Limitations of the intervention in this study suggested that participants and patients may have measurement biases. To improve the reliability and reference of the research, further improvements in research methods and design are needed.

Age, hyperlipidemia, smoking, diabetes mellitus, hypertension, and abdominal obesity were risk factors for death of cerebrovascular disease. This finding was consistent with the research results of Zuo et al. (16). In patients with cerebrovascular disease, thickened blood vessels cause cerebral hypoxia and a series of hematologic complications. Increased blood viscosity and abnormal platelet function tend to cause vascular stenosis and increased blood flow resistance (17-20). Active proteins protect cells, including by suppressing inflammation, maintaining endothelium, and the blood-brain barrier. Plasma levels of active protein $\mathrm{C}$ are associated with a reduced risk for cerebrovascular disease in patients with diabetes, especially in those with an increased risk of death from atherosclerosis caused by obesity. In patients with heart failure, blood stasis is caused by arterial endothelial insufficiency and venous dysfunction, pulmonary hypertension. There is an increased venous stagnation in the lower extremities, as well as the formation of thrombus in the heart chambers and blockage of blood vessels, endangering brain function. Researchers found a high incidence of cerebrovascular disease in overweight, alcohol drinking, and smoking patients $(21,22)$. Infection can directly damage new blood vessels and release inflammatory mediators (23). Inflammation of blood vessel walls can be combined by activation of common methods (For example: Hormonal disorders, male and smoking, and so on) to cause thrombosis, inflammation, and blood clotting, which is triggered by tissue factors. In addition, inflammation can directly activate the immune system and lead to blood clotting, eventually causing the death of patients with cerebrovascular disease $(24,25)$. In summary, early death from cerebrovascular disease is associated with multifactorial factors such as hypertension, death risk factors, hyperlipidemia, family history of stroke, diabetes, overweight, and alcohol consumption, and smoking are important risk factors for cardiovascular and cerebrovascular diseases. Death accounts for a relatively high proportion, and active intervention should be taken to reduce the early mortality rate of cerebrovascular diseases while curing the original disease and timely prevention and treatment of complications. Residents can be educated on safety, and everyone should be encouraged to adopt a low-sugar and low-fat diet to avoid hypertension, diabetes, hyperlipidemia, and overweight. Smoking and drinking also need to be controlled. Patients with a family history of stroke need to go to the hospital for physical examination, so as to rule out the possibility of early pathological changes in the brain nerves.

In this study, a lot of foreign literature was searched to study various risk factors. Because there was no comparison between the control and treatment groups, relevant Chinese literature was not included. The treatment time of the 10 included studies was contradictory, which may have a certain influence on the results of this meta-analysis. Moreover, randomization methods were not reported. Therefore, it is recommended that trial plans be further improved to standardize the specific time, method, and drugs used in periodic interventions. High-quality, largesample, and multicenter RCTs should be conducted to obtain more reliable evidence.

\section{Conclusions}

Patients with diabetes, hyperlipidemia, smoking, alcohol drinking, hypertension, and abdominal obesity combined with cerebrovascular disease had high mortality. The results of the meta-analysis were basically stable, but there were still limitations. Due to the limitation of different retrieval mechanisms, the retrieval may not be comprehensive. There were differences in risk factor assessment or measurement in the different studies, which may affect the results. All the studies included in the meta-analysis were from the published literature, and the failure to include unpublished studies may lead to potential publication bias. Therefore, it is suggested more high-quality, multicenter, and large-sample original studies be performed for verification in the future, so as to provide early warning for improvements in clinical practice and prevention of death from cerebrovascular diseases in the emergency neurology department.

\section{Acknowledgments}

Funding: None. 


\section{Footnote}

Reporting Checklist: The authors have completed the PRISMA reporting checklist. Available at https://dx.doi. org/10.21037/apm-21-2329

Conflicts of Interest: All authors have completed the ICMJE uniform disclosure form (available at https://dx.doi. org/10.21037/apm-21-2329). The authors have no conflicts of interest to declare.

Ethical Statement: The authors are accountable for all aspects of the work in ensuring that questions related to the accuracy or integrity of any part of the work are appropriately investigated and resolved.

Open Access Statement: This is an Open Access article distributed in accordance with the Creative Commons Attribution-NonCommercial-NoDerivs 4.0 International License (CC BY-NC-ND 4.0), which permits the noncommercial replication and distribution of the article with the strict proviso that no changes or edits are made and the original work is properly cited (including links to both the formal publication through the relevant DOI and the license). See: https://creativecommons.org/licenses/by-nc-nd/4.0/.

\section{References}

1. Yuan P, Xiang J, Borg M, et al. Analysis of lifetime death probability for major causes of death among residents in China. BMC Public Health 2020;20:1090.

2. Nakanishi M, Ogawa A, Nishida A. Availability of home palliative care services and dying at home in conditions needing palliative care: A population-based death certificate study. Palliat Med 2020;34:504-12.

3. Rolfs A, Fazekas F, Grittner U, et al. Acute cerebrovascular disease in the young: the Stroke in Young Fabry Patients study. Stroke 2013;44:340-9.

4. Barthels D, Das H. Current advances in ischemic stroke research and therapies. Biochim Biophys Acta Mol Basis Dis 2020;1866:165260.

5. Sarikaya H, Ferro J, Arnold M. Stroke prevention-medical and lifestyle measures. Eur Neurol 2015;73:150-7.

6. Zheng Q, Li Y, Zhang L, et al. Association between drinking and all-cause mortality in patients with ischemic stroke. Nan Fang Yi Ke Da Xue Xue Bao 2019;39:422-7.

7. Larsson SC, Wallin A, Wolk A, et al. Differing association of alcohol consumption with different stroke types: a systematic review and meta-analysis. BMC Med 2016;14:178.

8. Hatanaka Y, Shimokata K, Osugi S, et al. Impact of drinking and smoking habits on cerebrovascular disease risk among male employees. Sangyo Eiseigaku Zasshi 2016;58:155-63.

9. Alebeek ME, Arntz RM, Ekker MS, et al. Risk factors and mechanisms of stroke in young adults: The FUTURE study. J Cereb Blood Flow Metab 2018;38:1631-41.

10. Li L, Yiin GS, Geraghty OC, et al. Incidence, outcome, risk factors, and long-term prognosis of cryptogenic transient ischaemic attack and ischaemic stroke: a population-based study. Lancet Neurol 2015;14:903-13.

11. Edwards JD, Kapral MK, Lindsay MP, et al. Young Stroke Survivors With No Early Recurrence at High LongTerm Risk of Adverse Outcomes. J Am Heart Assoc 2019;8:e010370.

12. Bowers DC, Liu Y, Leisenring W, et al. Late-occurring stroke among long-term survivors of childhood leukemia and brain tumors: a report from the Childhood Cancer Survivor Study. J Clin Oncol 2006;24:5277-82.

13. MacClellan LR, Giles W, Cole J, et al. Probable migraine with visual aura and risk of ischemic stroke: the stroke prevention in young women study. Stroke 2007;38:2438-45.

14. O'Donnell MJ, Xavier D, Liu L, et al. Risk factors for ischaemic and intracerebral haemorrhagic stroke in 22 countries (the INTERSTROKE study): a case-control study. Lancet 2010;376:112-23.

15. Topcuoglu MA, Saka E, Silverman SB, et al. Recrudescence of Deficits After Stroke: Clinical and Imaging Phenotype, Triggers, and Risk Factors. JAMA Neurol 2017;74:1048-55.

16. Zuo L, Zhan Y, Liu F, et al. Clinical and laboratory factors related to acute isolated vertigo or dizziness and cerebral infarction. Brain Behav 2018;8:e01092.

17. NanZhu Y, Xin L, Xianghua Y, et al. Risk factors analysis of nosocomial pneumonia in elderly patients with acute cerebral infraction. Medicine (Baltimore) 2019;98:e15045.

18. Dupont SA, Dispenzieri A, Mauermann ML, et al. Cerebral infarction in POEMS syndrome: incidence, risk factors, and imaging characteristics. Neurology 2009;73:1308-12.

19. Pase MP, Himali JJ, Beiser AS, et al. Sugar- and Artificially Sweetened Beverages and the Risks of Incident Stroke and Dementia: A Prospective Cohort Study. Stroke 2017;48:1139-46.

20. Love S, Miners JS. Cerebrovascular disease in ageing and 
Alzheimer's disease. Acta Neuropathol 2016;131:645-58.

21. Mo J, Huang L, Peng J, et al. Autonomic Disturbances in Acute Cerebrovascular Disease. Neurosci Bull 2019;35:133-44.

22. Yong WC, Sanguankeo A, Upala S. Association between primary Sjögren's syndrome, cardiovascular and cerebrovascular disease: a systematic review and metaanalysis. Clin Exp Rheumatol 2018;36 Suppl 112:190-7.

23. Tong X, Yang Q, Ritchey MD, et al. The Burden of Cerebrovascular Disease in the United States. Prev Chronic Dis 2019;16:E52.

Cite this article as: Zhang Y, Yu H, Ke C, Sun Q. Systematic review and meta-analysis of various risk factors of death from emergency cerebrovascular diseases in the department of neurology. Ann Palliat Med 2021;10(10):10661-10673. doi: 10.21037/apm-21-2329
24. Toledo JB, Arnold SE, Raible K, et al. Contribution of cerebrovascular disease in autopsy confirmed neurodegenerative disease cases in the National Alzheimer's Coordinating Centre. Brain 2013;136:2697-706.

25. Kindgen-Milles D, Müller E, Buhl R, et al. Nasalcontinuous positive airway pressure reduces pulmonary morbidity and length of hospital stay following thoracoabdominal aortic surgery. Chest 2005;128:821-8.

(English Language Editor: K. Brown) 